\title{
Ventilation of intubated patients during HEMS hoisting operations
}

\author{
John HollottiD
}

\begin{abstract}
In response to the review "Advanced airway management in hoist and longline operations in mountain HEMS - considerations in austere environments: a narrative review." by Pietsch et al. we refer to recently published original research describing manual versus automatic ventilation of intubated patients during helicopter hoisting operations.
\end{abstract}

Keywords: Helicopters, Intratracheal intubation, Prehospital emergency care, Pulmonary ventilation, Rescue stretchers

\section{Main text}

The recently published "Advanced airway management in hoist and longline operations in mountain HEMS considerations in austere environments: a narrative review." [1] by Pietsch et al. is an excellent review of the issues involved in the helicopter hoisting of critically unwell patients, concentrating on alpine environments.

Contemporaneously to the authors' review we have published an original research paper "Ventilatory choices for intubated patients during helicopter stretcher winching" [2]. Concentrating on the method of ventilation, we compared the use of a self-inflating bag versus a mechanical ventilator during the helicopter hoisting (or winching) of a manikin, measuring airway pressures. The use of automatic ventilation was found to be more reliable, consistent and safer. As pointed out in Pietsch's article, airway monitoring and disconnection is a major concern, but of a low risk if managed appropriately, and the safety benefits to the patient make it potentially the optimal method. In addition, the enhancement of situational awareness for the attendant when freed from the task of ventilation is likely to particularly benefit operational safety.

Our paper appears to be the only publication to date which has directly addressed the safety of ventilation during hoist operations. We believe that we have been able to demonstrate the advantages to both patient and team of utilising mechanical ventilation.

\section{Abbreviations}

HEMS: Helicopter Emergency Medical Services; ICAR MEDCOM: International Commission for Mountain Emergency Medicine

\section{Availability of data and supporting materials section \\ The datasets used and analysed during the study referred to are available from} the corresponding author on reasonable request.

\section{Authors' contributions}

$\mathrm{JH}$ designed and wrote the study referred to and authored the letter to the editor. The author read and approved the final manuscript.

Ethics approval and consent to participate

The Hunter New England Human Research Ethics Committee granted approval to undertake the study referred to.

\section{Competing interests}

The author declares that they have no competing interests.

\section{Publisher's Note}

Springer Nature remains neutral with regard to jurisdictional claims in published maps and institutional affiliations.

Received: 3 May 2018 Accepted: 7 May 2018

Published online: 11 May 2018

\section{References \\ 1. Pietsch U, Knapp J, Kreuzer $\mathrm{O}$, et al. Advanced airway management in hoist and longline operations in mountain HEMS - considerations in austere environments: a narrative review this review is endorsed by the International Commission for Mountain Emergency Medicine (ICAR MEDCOM). Scand J Trauma Resusc Emerg Med. 2018:26:23-30. \\ 2. Hollott J. Ventilatory choices for intubated patients during helicopter stretcher winching. Emerg Med Aust. 2017;29:692-6.}

